\title{
Is abnormal myocardial repolarization associated with the occurrence of malignant tachyarrhythmias in Takotsubo cardiomyopathy?
}

\author{
Florian Streitner ${ }^{1 *}$, Karsten Hamm ${ }^{1 *}$, Ilan S. Wittstein ${ }^{2}$, Adrian Baranchuk ${ }^{3}$, \\ Yoshihiro J. Akashi ${ }^{4}$, Holger M. Nef ${ }^{5}$, Laurent Bonello ${ }^{6}$, Christian Wolpert ${ }^{7}$, \\ Martin Borggrefe ${ }^{1}$, Dariusch Haghi ${ }^{1}$ \\ ${ }^{1} 1^{\text {st }}$ Department of Medicine-Cardiology, University Medical Centre Mannheim, Mannheim, Germany \\ ${ }^{2}$ Division of Cardiology, Department of Medicine, Johns Hopkins University \\ School of Medicine, Baltimore, USA \\ ${ }^{3}$ Division of Cardiology, Kingston General Hospital, Kingston, Ontario, Canada \\ ${ }^{4}$ Division of Cardiology, Department of Internal Medicine, St. Marianna University \\ School of Medicine, Kawasaki-City, Japan \\ ${ }^{5}$ Department of Cardiology, Kerckhoff Heart and Thorax Center, Bad Nauheim, Germany \\ ${ }^{6}$ Department of Cardiology, Hopital Universitaire Nord, Marseille, France \\ ${ }^{7}$ Department of Cardiology, Ludwigsburg General Hospital, Ludwigsburg, Germany
}

\begin{abstract}
Background: Abnormalities of cardiac repolarization are a hallmark of Takotsubo cardiomyopathy (TC), but their association with the occurrence of syncope and ventricular tachyarrhythmias is unknown. This study sought to assess the relationship between myocardial repolarization and malignant tachyarrhythmias in $T C$.

Methods: Clinical data and electrocardiographic repolarization parameters of 28 patients with TC and ventricular tachyarrhythmias $(n=26)$ or syncope $(n=2)$ were compared to data from 20 randomly selected patients with TC but without ventricular tachyarrhythmias or syncope.

Results: Study patients had significantly lower ejection fraction (EF) compared with controls $(35 \pm 14 \%$ vs. $46 \pm 10 \%, p=0.006)$. On day 1 , no significant differences in repolarization $p a$ rameters were observed. However, in the subgroup with ventricular fibrillation ([VF]; $n=10$ ), Tpeak-Tend in lead V6 was significantly prolonged (97 \pm 20 vs. $85 \pm 19 \mathrm{~ms} ; p=0.04)$. Similarly, in the subgroup with torsade de pointes ([TdP]; $n=5)$ Tpeak-Tend in lead V4 was prolonged (127 \pm 21 vs. $94 \pm 27 \mathrm{~ms} ; p=0.001)$. On day 3, Tpeak-Tend in lead V3 (130 \pm \pm 51 vs. $105 \pm 21 \mathrm{~ms}, p=0.049)$ and Tpeak-Tend dispersion (56 \pm 33 vs. $36 \pm 21 \mathrm{~ms}$; $p=0.03$ ) were significantly longer in study patients. The difference in Tpeak-Tend in lead V3 was borderline in the VF subgroup, but significant in the subgroup with TdP. The latter group had also longer Tpeak-Tend in lead V4 and longer corrected QT interval in leads V3 and V4. Conclusions: Patients with TC who experience malignant tachyarrhythmias have lower $E F$ and a more pronounced alteration of the spatial dispersion of ventricular repolarization. (Cardiol J 2013; 20, 6: 633-638)
\end{abstract}

Key words: Takotsubo cardiomyopathy, ventricular tachyarrhythmia, ventricular repolarization

Address for correspondence: Florian Streitner, MD, $1^{\text {st }}$ Department of Medicine-Cardiology, University Medical Centre Mannheim, Theodor-Kutzer-Ufer 1-3, 68167 Mannheim, Germany, tel: +49 621 383 5239, fax: +49 6213832172 , e-mail:kontakt@kardiologie-weinheim.de

*These authors contributed equally to this work.

Received: 17.01.2013 Accepted: 09.04.2013 


\section{Introduction}

Takotsubo cardiomyopathy (TC) is characterized by transient left-ventricular wall motion abnormalities unrelated to significant coronary artery disease along with ECG abnormalities and/ /or elevation of cardiac troponin. Syncope and life-threatening ventricular tachyarrhythmias are rare but well known complications of the syndrome [1, 2]. However, little is known about the risk factors that could lead to the development of malignant tachyarrhythmias. Several studies have found characteristic abnormalities of the ventricular repolarization [3, 4]. Whether there is an association between abnormal repolarization and occurrence of life-threatening tachyarrhythmias in TC remains unknown.

The aim of this study was to investigate the association of various parameters of myocardial repolarization with the development of malignant tachyarrhythmias in patients with TC.

\section{Methods}

Among 73 consecutive patients with a clinical diagnosis of TC at the University Medical Center Mannheim (UMCM), 9 were identified who either had an episode of ventricular tachyarrhythmia $(\mathrm{n}=7)$ or whose initial presentation was an episode of syncope $(n=2)$. In January 2008 we also contacted 7 authors who had previously published cases or case series with ventricular tachyarrhythmias in TC to contribute data from their published and, if available, from additional unpublished patients to the study. Overall, 5 authors contributed data from 20 patients with TC and malignant ventricular tachyarrhythmias [5-9]. Of these, 1 patient was excluded because of poor ECG quality that prohibited further analysis. Finally, a total of 28 patients comprised the study cohort (group 1). Among the remaining 64 patients at the UMCM without ventricular tachyarrhythmias, 20 were randomly selected and served as controls (group 2).

Diagnosis of TC was based on the Mayo Clinic criteria [10]: 1) Acute onset of mid-left-ventricular wall-motion abnormalities with or without apical involvement and not confined to the vascular territory of a single major coronary artery; 2) Absence of obstructive coronary disease or angiographic evidence of plaque rupture; 3) New ECG abnormalities (either ST-segment elevation and/or T-wave inversion) or elevated cardiac troponin in the absence of pheochromocytoma, and myocarditis.

Resting 12-lead ECGs $(50 \mathrm{~mm} / \mathrm{s}, 1 \mathrm{mV} / \mathrm{mm}$ in German centers and $25 \mathrm{~mm} / \mathrm{s}, 1 \mathrm{mV} / \mathrm{mm}$ in all other centers) on day 1 (day of the initial diagnosis) and day 3 were used to assess ventricular repolarisation. All measurements were performed manually and blinded to the patients' clinical data. The QT intervals in each of the 12 leads were measured from the beginning of the QRS complex to the visual return of the T-wave to the isoelectric line for every patient. Where the T-wave was complicated by a U-wave, the end of the T-wave was defined as the nadir between the $\mathrm{T}$ - and $\mathrm{U}$-wave [11]. T-waves smaller than $1 \mathrm{~mm}$ in amplitude were not measured. The QT intervals were corrected for heart rate (QTc) using Bazett's formula. The QT dispersion (QTD) for each ECG recording was defined as the difference between the longest and shortest measured QT [12]. The Tpeak intervals (Tp) in each of the 6 precordial leads were measured from the beginning of the QRS complex to the peak of the $\mathrm{T}$ wave (if the $\mathrm{T}$ wave was positive) or to the nadir of the $\mathrm{T}$ wave (if the $\mathrm{T}$ wave was negative or biphasic). The Tpeak-to-end interval (Tp-e) was derived from the equation: QT interval - Tp interval. The $\mathrm{Tp}$-e dispersion (Tp-eD) was defined as the difference between the longest and shortest measured Tp-e [13]. All measurements were averaged for 2 consecutive beats in patients with sinus rhythm and for 3 consecutive beats in atrial fibrillation. Intra-observer and inter-observer variability was assessed in 20 randomly selected ECGs.

Review of these data was approved by the local review board.

\section{Statistical analysis}

Continuous variables are expressed as mean \pm \pm standard deviation. Continuous variables were compared using the unpaired student $t$ test or Mann-Whitney $U$ test. Categorial variables were compared with the $\chi^{2}$ test or Fisher exact test. To assess colinearity or independency of lower left ventricular ejection fraction (EF) and Tp-e variables, Spearman's rank correlation coefficients were calculated. A p value $<0.05$ was considered statistically significant.

\section{Results}

The clinical features of patients are shown in Table 1. Baseline parameters were similar between both groups except for EF which was significantly lower in patients with malignant tachyarrhythmias $(35 \pm 14 \%$ vs. $46 \pm 10 \%, p=0.006)$. Overall, the following tachyarrhythmias were observed: Non-sustained ventricular tachycardia in 8 , ventricular tachycardia (VT) in 3, ventricular fibrillation (VF) in 4 , torsade de pointes (TdP) in 4 , both VT and 
Table 1. Baseline characteristics.

\begin{tabular}{lccc}
\hline & Group 1 & Group 2 & P \\
\hline Age [years] & $63 \pm 15$ & $67 \pm 11$ & 0.37 \\
Women & $24(86 \%)$ & $19(95 \%)$ & 0.38 \\
Men & $4(14 \%)$ & $1(5 \%)$ & \\
Variant of Takotsubo cardiomyopathy: & & & \\
$\quad$ Apical & $25(89 \%)$ & $15(75 \%)$ & 0.25 \\
$\quad$ Midventricular & $2(7 \%)$ & $5(25 \%)$ & 0.11 \\
$\quad$ Basal & $1(4 \%)$ & $0(0 \%)$ & 1 \\
Troponin $[\mu \mathrm{g} / \mathrm{L}]$ & $5.8 \pm 6.6$ & $3.2 \pm 4$ & 0.13 \\
Ejection fraction [\%] & $35 \pm 14$ & $46 \pm 10$ & 0.006 \\
Survival to discharge & $26(93 \%)$ & $20(100 \%)$ & 0.37 \\
\hline
\end{tabular}

$\mathrm{VF}$ in 6 and both VT and TdP in 1 patient. Events occurred on day 1 in 24 patients (86\%). In 1 patient, episodes of VT and VF occurred on days 4, 7 and 8. One patient had an episode of TdP on day 6 and recurrent episodes of VT on day 9. Non-sustained ventricular tachycardia occurred on day 4 in $1 \mathrm{pa}$ tient and on day 5 in another.

QTc increased significantly from day 1 to day 3 , both in group $1(450 \pm 32$ vs. $487 \pm 52 \mathrm{~ms}, \mathrm{p}=$ $=0.004)$ and in group $2(449 \pm 31 \mathrm{vs} .485 \pm 33 \mathrm{~ms}$, p < 0.001) as did Tp-e (group 1: $89 \pm 18$ vs. $121 \pm$ $\pm 38 \mathrm{~ms}, \mathrm{p}<0.001$; group $2: 82 \pm 16$ vs. $142 \pm 28 \mathrm{~ms}$, $\mathrm{p}<0.001)$. Heart rate decreased significantly from day 1 to day 3 (group 1: $84 \pm 19$ vs. $74 \pm 15 \mathrm{bpm}$, $\mathrm{p}=0.04$; group $2: 99 \pm 16$ vs. $85 \pm 20 \mathrm{bpm}$, $\mathrm{p}=0.02)$. QTD and Tp-eD did not change significantly from day 1 to day 3 . Intra-observer and inter-observer variability were $15 \pm 31$ and $19 \pm$ $\pm 33 \mathrm{~ms}$, respectively. Combined Tp-e values and Tp-e values within groups at day 1 and day 3 did not reveal any correlation with $\mathrm{EF}$ (each $\mathrm{p}=\mathrm{NS})$.

\section{Repolarization parameters on day 1}

On day 1 , no significant differences were observed for various repolarization parameters between group 1 and group 2 (Table 2). However, when only patients with an episode of VF $(n=10)$ were analyzed, Tp-e in lead V6 was significantly prolonged (Table 3). Similarly, subgroup analysis of patients with $\operatorname{TdP}(n=5)$ revealed significantly greater Tp-e in lead V4 in this subset of patients (Table 3).

Compared with controls, heart rates were significantly lower in group 1 patients (Table 2). This was predominantly caused by low heart rates in the subgroup of patients with $\mathrm{TdP}$ whose heart rates were significantly lower than heart rates in the remainder of group 1 ( $67 \pm 12$ vs. $87 \pm 18 \mathrm{~ms}$, $\mathrm{p}=0.03)$ or heart rates in group $2(67 \pm 12$ vs. $99 \pm 16, \mathrm{p}<0.001)$.

\section{Repolarization parameters on day 3}

On day 3 , there were significant differences between group 1 and group 2 with regard to heart rate, Tp-e in lead V3 and Tp-eD (Table 2). Subgroup analysis of patients with VF revealed no significant differences for heart rate and borderline significance for Tp-e in lead V3. Tp-eD was significantly higher in group 1. Subgroup analysis of patients with TdP demonstrated significant differences for several parameters including heart rate, QTc in lead aVL, QTc in lead V3, QTc in lead V4, Tp-e in lead V3 and Tp-e in lead V4 (Table 3).

\section{Discussion}

Abnormalities of cardiac repolarization are a hallmark of TC and were observed as early as 1990 when, for the first time, Sato et al. [14] reported on this disease entity. Despite this, the incidence of malignant ventricular arrhythmias in TC seems to be rather low ranging from $1 \%$ to $9 \%$ in several large series of patients with the disease [15-18].

Previous reports have shown characteristic changes in the 12-lead ECG in TC. Matsuoka et al. [4] examined the time course of the ECG in 10 consecutive patients. All patients had ST segment elevation upon presentation and developed T-wave inversions in several leads on the following days ("subacute phase"). Takashio et al. [19] reported that the electrocardiographic extent and magnitude of ST-segment elevation are potentially useful predictors of in-hospital complications in patients presenting with TC.

Maximum QT interval, minimum QT interval, QTc, QTD, heart rate-corrected QTD, and Tp-e 
Table 2. Repolarisation parameters of the entire study population.

\begin{tabular}{|c|c|c|c|c|c|c|}
\hline \multirow[t]{2}{*}{ Parameter } & \multicolumn{3}{|c|}{ Day 1} & \multicolumn{3}{|c|}{ Day 3} \\
\hline & $\begin{array}{l}\text { Group } 1 \\
(\mathrm{n}=28)\end{array}$ & $\begin{array}{l}\text { Group } 2 \\
(\mathrm{n}=20)\end{array}$ & $\mathbf{P}$ & $\begin{array}{l}\text { Group } 1 \\
(\mathrm{n}=28)\end{array}$ & $\begin{array}{l}\text { Group } 2 \\
(\mathrm{n}=20)\end{array}$ & $\mathbf{P}$ \\
\hline Heart rate $[\mathrm{bpm}]$ & $84 \pm 19$ & $99 \pm 16$ & 0.009 & $74 \pm 15$ & $85 \pm 21$ & 0.048 \\
\hline QTc I [ms] & $441 \pm 41$ & $443 \pm 27$ & 0.83 & $492 \pm 52$ & $488 \pm 39$ & 0.76 \\
\hline QTc II [ms] & $447 \pm 39$ & $442 \pm 39$ & 0.73 & $476 \pm 59$ & $477 \pm 41$ & 0.99 \\
\hline QTc III [ms] & $422 \pm 98$ & $443 \pm 45$ & 0.42 & $487 \pm 55$ & $475 \pm 46$ & 0.48 \\
\hline QTc aVR [ms] & $454 \pm 39$ & $444 \pm 33$ & 0.39 & $489 \pm 55$ & $490 \pm 46$ & 0.97 \\
\hline QTc aVL [ms] & $441 \pm 45$ & $443 \pm 49$ & 0.92 & $496 \pm 54$ & $471 \pm 43$ & 0.12 \\
\hline QTc aVF [ms] & $452 \pm 40$ & $448 \pm 42$ & 0.72 & $474 \pm 59$ & $467 \pm 37$ & 0.69 \\
\hline QTc V1 [ms] & $439 \pm 50$ & $447 \pm 45$ & 0.57 & $466 \pm 54$ & $477 \pm 46$ & 0.52 \\
\hline QTc V2 [ms] & $439 \pm 39$ & $456 \pm 39$ & 0.17 & $490 \pm 65$ & $494 \pm 43$ & 0.78 \\
\hline QTc V3 [ms] & $452 \pm 37$ & $451 \pm 33$ & 0.92 & $499 \pm 70$ & $492 \pm 43$ & 0.71 \\
\hline QTc V4 [ms] & $454 \pm 38$ & $453 \pm 41$ & 0.90 & $513 \pm 62$ & $502 \pm 35$ & 0.5 \\
\hline QTc V5 [ms] & $458 \pm 38$ & $458 \pm 34$ & 0.99 & $496 \pm 63$ & $496 \pm 38$ & 0.99 \\
\hline QTc V6 [ms] & $458 \pm 33$ & $445 \pm 31$ & 0.20 & $494 \pm 65$ & $484 \pm 39$ & 0.56 \\
\hline QTc mean [ms] & $450 \pm 32$ & $448 \pm 31$ & 0.87 & $449 \pm 75$ & $485 \pm 32$ & 0.09 \\
\hline QT dispersion [ms] & $66 \pm 29$ & $60 \pm 23$ & 0.37 & $79 \pm 44$ & $65 \pm 26$ & 0.24 \\
\hline Tp-e V1 [ms] & $79 \pm 23$ & $80 \pm 24$ & 0.80 & $118 \pm 43$ & $96 \pm 46$ & 0.15 \\
\hline Tp-e V2 [ms] & $87 \pm 23$ & $93 \pm 27$ & 0.81 & $118 \pm 40$ & $109 \pm 42$ & 0.47 \\
\hline Tp-e V3 [ms] & $84 \pm 17$ & $94 \pm 27$ & 0.14 & $130 \pm 51$ & $105 \pm 21$ & 0.049 \\
\hline Tp-e V4 [ms] & $85 \pm 17$ & $94 \pm 27$ & 0.21 & $131 \pm 42$ & $114 \pm 28$ & 0.12 \\
\hline Tp-e V5 [ms] & $83 \pm 20$ & $88 \pm 19$ & 0.22 & $123 \pm 48$ & $108 \pm 29$ & 0.22 \\
\hline Tp-e V6 [ms] & $78 \pm 22$ & $85 \pm 19$ & 0.17 & $120 \pm 42$ & $102 \pm 25$ & 0.08 \\
\hline Tp-e mean [ms] & $83 \pm 15$ & $97 \pm 21$ & 0.21 & $121 \pm 38$ & $141 \pm 28$ & 0.09 \\
\hline Tp-e dispersion [ms] & $36 \pm 16$ & $42 \pm 18$ & 0.25 & $56 \pm 33$ & $36 \pm 21$ & 0.041 \\
\hline
\end{tabular}

QTc — corrected QT interval; Tp-e — Tpeak-to-end interval

were all significantly prolonged during the "subacute phase" of the disease. Kurisu et al. [3] examined the time course of the ECG in 16 patients. They observed that the QT interval as well as the depth of the T-wave had a 2-peaked time course, one occurring around days $2-3$ and the other one occurring between days 14-21. Bonnemeier et al. [20], in a case control study, demonstrated that patients with TC, unlike patients with reperfused anterior myocardial infarction, continue to show modulation of the QT interval with changes in the heart rate.

So far, only one study has systematically analysed malignant arrhythmias in TC [21]. Out of 105 patients with TC at the Mayo Clinic, 6 were identified who had experienced either VF $(n=3)$ or significant bradyarrhythmias $(n=3)$. The study group was compared to 31 randomly selected control patients with TC but without arrhythmias. When compared with controls, the study patients had higher R-R interval variability (maximal R-R interval - minimal R-R interval in the first $24 \mathrm{~h}$ of admission) and longer QTc. PR interval was also longer in the study group, but the difference disappeared, once the PR interval was corrected for the heart rate. The authors also found that all patients in the study group had a history of atrial arrhythmias $(n=5)$ or atrio-ventricular nodal dysfunction $(\mathrm{n}=1)$. However, in that study the small number of patients and the heterogeneous nature of observed arrhythmias precluded firm conclusions.

To our knowledge, this is the largest series of patients with malignant tachyarhythmias in TC. Similar to what is known from many other cardiac diseases, patients with malignant tachyarrhythmias had significantly lower EF compared to controls. This difference remained significant even after excluding those 5 patients whose presenting symptom had been a cardiac arrest and who, therefore, might have had some degree of cardiac arrest-related myocardial stunning.

In accordance with previous reports, QTc increased significantly during the first few days following diagnosis of TC. In contradistinction to a previous report [21], however, mean QTc did not 
Table 3. Repolarization parameters by subgroups.

\begin{tabular}{|c|c|c|c|c|c|c|}
\hline \multirow[t]{2}{*}{ Parameter } & \multicolumn{3}{|c|}{ Day 1} & \multicolumn{3}{|c|}{ Day 3} \\
\hline & $\begin{array}{c}\text { VF } \\
(n=10)\end{array}$ & $\begin{array}{c}\text { TdP } \\
(n=5)\end{array}$ & $\begin{array}{l}\text { Group } 2 \\
(\mathrm{n}=20)\end{array}$ & $\begin{array}{c}\text { VF } \\
(n=10)\end{array}$ & $\begin{array}{c}\text { TdP } \\
(n=5)\end{array}$ & $\begin{array}{l}\text { Group } 2 \\
(n=20)\end{array}$ \\
\hline Heart rate $[\mathrm{bpm}]$ & $91 \pm 24$ & $63 \pm 6^{*}$ & $99 \pm 16$ & $81 \pm 17$ & $62 \pm 11 *$ & $85 \pm 21$ \\
\hline QTc I [ms] & $439 \pm 30$ & $453 \pm 60$ & $443 \pm 27$ & $480 \pm 62$ & $521 \pm 74$ & $488 \pm 39$ \\
\hline QTc II [ms] & $460 \pm 39$ & $449 \pm 51$ & $442 \pm 39$ & $484 \pm 62$ & $487 \pm 105$ & $477 \pm 41$ \\
\hline QTc III [ms] & $372 \pm 155$ & $445 \pm 27$ & $443 \pm 45$ & $482 \pm 63$ & $500 \pm 63$ & $475 \pm 46$ \\
\hline QTc aVR [ms] & $456 \pm 34$ & $460 \pm 43$ & $444 \pm 33$ & $480 \pm 53$ & $545 \pm 75$ & $490 \pm 46$ \\
\hline QTc aVL [ms] & $425 \pm 62$ & $450 \pm 30$ & $443 \pm 49$ & $501 \pm 66$ & $550 \pm 54^{*}$ & $471 \pm 43$ \\
\hline QTc aVF [ms] & $452 \pm 51$ & $466 \pm 57$ & $448 \pm 42$ & $461 \pm 61$ & - & $467 \pm 37$ \\
\hline QTc V1 [ms] & $433 \pm 42$ & $469 \pm 24$ & $447 \pm 45$ & $460 \pm 57$ & $510 \pm 68$ & $477 \pm 46$ \\
\hline QTc V2 [ms] & $439 \pm 39$ & $460 \pm 31$ & $456 \pm 39$ & $483 \pm 82$ & $525 \pm 76$ & $494 \pm 43$ \\
\hline QTc V3 [ms] & $455 \pm 39$ & $463 \pm 39$ & $451 \pm 33$ & $497 \pm 85$ & $566 \pm 28 *$ & $492 \pm 43$ \\
\hline QTc V4 [ms] & $464 \pm 38$ & $468 \pm 46$ & $453 \pm 41$ & $515 \pm 69$ & $569 \pm 34^{*}$ & $502 \pm 35$ \\
\hline QTc V5 [ms] & $459 \pm 41$ & $471 \pm 48$ & $458 \pm 34$ & $497 \pm 74$ & $539 \pm 60$ & $496 \pm 38$ \\
\hline QTc V6 [ms] & $465 \pm 36$ & $465 \pm 44$ & $445 \pm 31$ & $476 \pm 71$ & $533 \pm 84$ & $484 \pm 39$ \\
\hline QTc mean [ms] & $450 \pm 34$ & $461 \pm 36$ & $448 \pm 31$ & $481 \pm 55$ & $522 \pm 74$ & $485 \pm 32$ \\
\hline QT dispersion [ms] & $65 \pm 17$ & $60 \pm 37$ & $60 \pm 23$ & $86 \pm 57$ & $58 \pm 31$ & $65 \pm 26$ \\
\hline Tp-e V1 [ms] & $105 \pm 30$ & $75 \pm 7$ & $80 \pm 24$ & $120 \pm 50$ & $120 \pm 66$ & $96 \pm 46$ \\
\hline Tp-e V2 [ms] & $101+/-28$ & $75 \pm 39$ & $93 \pm 27$ & $125 \pm 51$ & $113 \pm 62$ & $109 \pm 42$ \\
\hline Tp-e V3 [ms] & $97 \pm 30$ & $95 \pm 44$ & $94 \pm 27$ & $136 \pm 60^{*}$ & $180 \pm 62^{*}$ & $105 \pm 21$ \\
\hline Tp-e V4 [ms] & $97 \pm 27$ & $127 \pm 21^{*}$ & $94 \pm 27$ & $136 \pm 45$ & $163 \pm 47^{*}$ & $114 \pm 28$ \\
\hline Tp-e V5 [ms] & $90 \pm 20$ & $95 \pm 26$ & $88 \pm 19$ & $138 \pm 52$ & $148 \pm 69$ & $108 \pm 29$ \\
\hline Tp-e V6 [ms] & $97 \pm 20^{*}$ & $88 \pm 15$ & $85 \pm 19$ & $118 \pm 50$ & $143 \pm 64$ & $102 \pm 25$ \\
\hline Tp-e mean [ms] & $95 \pm 27$ & $93 \pm 28$ & $97 \pm 21$ & $128 \pm 46$ & $134 \pm 64$ & $141 \pm 28$ \\
\hline Tp-e dispersion [ms] & $39 \pm 17$ & $50 \pm 22$ & $42 \pm 18$ & $59 \pm 32^{*}$ & $50 \pm 29$ & $36 \pm 21$ \\
\hline
\end{tabular}

${ }^{*} \mathrm{p}<0.05$ vs. Group 2; QTc - corrected QT interval; TdP — torsade de pointes; Tp-e - Tpeak-to-end interval; VF — ventricular fibrillation

differ significantly between study patients and controls. This was also true for the subset of patients with TdP in whom one would intuitively expect particularly long QTc intervals. Our findings concur with observations made by Samuelov-Kinori et al. [22]. In their recently published study, the authors compared 15 patients with TC-associated TdP from published reports with 86 patients from published reports who had TC-associated QT interval prolongation without TdP. They found that when compared to patients without TdP, patients with TdP had only a trend towards longer QTc intervals which did not reach statistical significance. Of note, although mean QTc in our study did not differ between patients and controls, QTc in several single leads (aVL, V3 and V4) was significantly longer in patients with TdP, indicating the possibility of significant regional heterogeneity of the QT interval in TC. This could be explained by a disease process that affects the myocardium in a non-uniform manner. Importantly, patients with TdP had significantly lower heart rates, when compared to other study patients or to controls, indicating a potential role of low heart rates in the occurrence of TdP.

Changes of the Tp-e interval are thought to be capable of reflecting changes in the spatial dispersion of repolarization, and thus may be prognostic of arrhythmic risk in a variety of different cardiac pathologies [23], including TdP during acquired bradyarrhythmias [24] and VT in coronary artery disease [25]. An extensive review of this concept has been published elsewhere [23]. As transmural dispersion of repolarization can be highly variable in different myocardial regions, it is important to analyse Tp-e in each of the precordial leads independently and not to average them across several leads [23]. By doing so, we found significant differences in single leads among different subgroups of patients and at different time points. For example, patients with TdP had prolonged Tp-e in lead V4 on day 1 and prolonged Tp-e in leads V3 and V4 on day 3, whereas patients with VF had only prolonged 
Tp-e in lead V6 on day 1 . This could indicate that patients with malignant arrhythmias have a more pronounced spatial dispersion of repolarization than patients who do not experience these types of arrhythmias. Additionally, the spatial dispersion of repolarization seems to be dynamic in nature.

QTD, which is believed to be a crude and indirect measure of general repolarization abnormalities [26], did not differ significantly between study patients and controls. However, QTD values in both groups seem to be higher than what has been reported in healthy individuals where QTD varies mostly between 30 and $60 \mathrm{~ms}$ [26].

\section{Limitations of the study}

This was a retrospective study, and thus some data relevant to malignant tachyarrhythmias may have not been collected at the time of diagnosis. Furthermore, the small number of patients in different subgroups limits the interpretation of subgroup data.

Manual measurements of repolarization intervals can be unreliable. This is particularly due to the uncertainties associated with the manual determination of the T-wave offset. The number of measurable leads in the 12-lead ECG can also significantly influence the range of QT and Tp-e interval durations, and thus can be a significant source of error.

\section{Conclusions}

In summary, patients with $\mathrm{TC}$ who experience malignant tachyarrhythmias have lower EF and a more pronounced alteration of the spatial dispersion of ventricular repolarization. Whether or not there is a true cause-effect relationship between abnormal repolarization and occurrence of malignant tachyarrhytmias in TC, however, remains to be seen.

\section{Conflict of interest: none declared}

\section{References}

1. Gianni M, Dentali F, Grandi AM et al. Apical ballooning syndrome or takotsubo cardiomyopathy: A systematic review. Eur Heart J, 2006; 27: 1523-1529.

2. Bybee KA, Kara T, Prasad A et al. Systematic review: transient left ventricular apical ballooning: A syndrome that mimics ST-segment elevation myocardial infarction. Ann Intern Med, 2004; 141: 858-865.

3. Kurisu S, Inoue I, Kawagoe T et al. Time course of electrocardiographic changes in patients with tako-tsubo syndrome: Comparison with acute myocardial infarction with minimal enzymatic release. Circ J, 2004; 68: 77-81.

4. Matsuoka K, Okubo S, Fujii E et al. Evaluation of the arrhythmogenecity of stress-induced "Takotsubo cardiomyopathy" from the time course of the 12-lead surface electrocardiogram. Am J Cardiol, 2003; 92: 230-233.
5. Akashi YJ, Nakazawa K, Kida K et al. Reversible ventricular dysfunction (takotsubo cardiomyopathy) following polymorphic ventricular tachycardia. Can J Cardiol, 2003; 19: 449-451.

6. Bonello L, Com O, Ait-Moktar O et al. Ventricular arrhythmias during Tako-tsubo syndrome. Int J Cardiol, 2008; 128: e50$-\mathrm{e} 53$.

7. Nault MA, Baranchuk A, Simpson CS et al. Takotsubo cardiomyopathy: A novel "proarrhythmic" disease. Anadolu Kardiyol Derg, 2007; 7 (Suppl. 1): 101-103.

8. Nef HM, Mollmann H, Kostin S et al. Tako-Tsubo cardiomyopathy: Intraindividual structural analysis in the acute phase and after functional recovery. Eur Heart J, 2007; 28: 2456-2464.

9. Wittstein IS, Thiemann DR, Lima JA et al. Neurohumoral features of myocardial stunning due to sudden emotional stress. N Engl J Med, 2005; 352: 539-548.

10. Prasad A, Lerman A, Rihal CS. Apical ballooning syndrome (Tako-Tsubo or stress cardiomyopathy): A mimic of acute myocardial infarction. Am Heart J, 2008; 155: 408-417.

11. Brendorp B, Elming H, Jun L et al. Qt dispersion has no prognostic information for patients with advanced congestive heart failure and reduced left ventricular systolic function. Circulation, 2001; 103: 831-835.

12. Day CP, McComb JM, Campbell RW. QT dispersion: an indication of arrhythmia risk in patients with long QT intervals. Br Heart J, 1990; 63: 342-344.

13. Castro Hevia J, Antzelevitch C, Tornes Barzaga F et al. Tpeak-Tend and Tpeak-Tend dispersion as risk factors for ventricular tachycardia/ventricular fibrillation in patients with the Brugada syndrome. J Am Coll Cardiol, 2006; 47: 1828-1834.

14. Sato H, Tateishi H, Uchida T. Takotsubo-type cardiomyopathy due to multivessel spasm. In: Kodama K, Haze K, Hon M eds. Clinical aspect of myocardial injury: From ischemia to heart failure [in Japanese]. $1^{\text {st }}$ Ed. Kagakuhyouronsha Publishing Co.; Tokyo 1990: $56-64$.

15. Eshtehardi P, Koestner SC, Adorjan P et al. Transient apical ballooning syndrome: Clinical characteristics, ballooning pattern, and long-term follow-up in a Swiss population. Int J Cardiol, 2009; 135: 370-375.

16. Regnante RA, Zuzek RW, Weinsier SB et al. Clinical characteristics and four-year outcomes of patients in the Rhode Island Takotsubo Cardiomyopathy Registry. Am J Cardiol, 2009; 103: 1015-1019.

17. Tsuchihashi K, Ueshima K, Uchida $\mathrm{T}$ et al. Transient left ventricular apical ballooning without coronary artery stenosis: a novel heart syndrome mimicking acute myocardial infarction. Angina Pectoris-Myocardial Infarction Investigations in Japan. J Am Coll Cardiol, 2001; 38: 11-18.

18. Syed FF, Asirvatham SJ, Franci J. Arrhythmia occurrence with takotsubo cardiomyopathy: a literature review. Europace, 2011; 13: 780-788.

19. Takashio S, Yamamuro M, Kojima S et al. Usefulness of sum of ST-segment elevation on electrocardiograms (Limb Leads) for predicting in-hospital complications in patients with stress (Takotsubo) cardiomyopathy. Am J Cardiol, 2012; 109: 1651-1656.

20. Bonnemeier H, Ortak J, Bode F et al. Modulation of ventricular repolarization in patients with transient left ventricular apical ballooning: A case control study. J Cardiovasc Electrophysiol, 2006; 17: 1340-1347.

21. Dib C, Prasad A, Friedman PA et al. Malignant arrhythmia in apical ballooning syndrome: Risk factors and outcomes. Indian Pacing Electrophysiol J, 2008; 8: 182-192.

22. Samuelov-Kinori L, Kinori M, Kogan Y et al. Takotsubo cardiomyopathy and QT interval prolongation: who are the patients at risk for torsades de pointes? J Electrocardiol, 2009; 42: 353-357 e351.

23. Antzelevitch C. Drug-induced spatial dispersion of repolarization. Cardiol J, 2008; 15: 100-121.

24. Topilski I, Rogowski O, Rosso R et al. The morphology of the QT interval predicts torsade de pointes during acquired bradyarrhythmias. J Am Coll Cardiol, 2007; 49: 320-328.

25. Lubinski A, Kornacewicz-Jach Z, Wnuk-Wojnar AM et al. The terminal portion of the $T$ wave: a new electrocardiographic marker of risk of ventricular arrhythmias. Pacing Clin Electrophysiol, 2000; 23(11 Part 2):1957-1959.

26. Malik M, Batchvarov VN. Measurement, interpretation and clinical potential of QT dispersion. J Am Coll Cardiol, 2000; 36: 1749-1766. 